
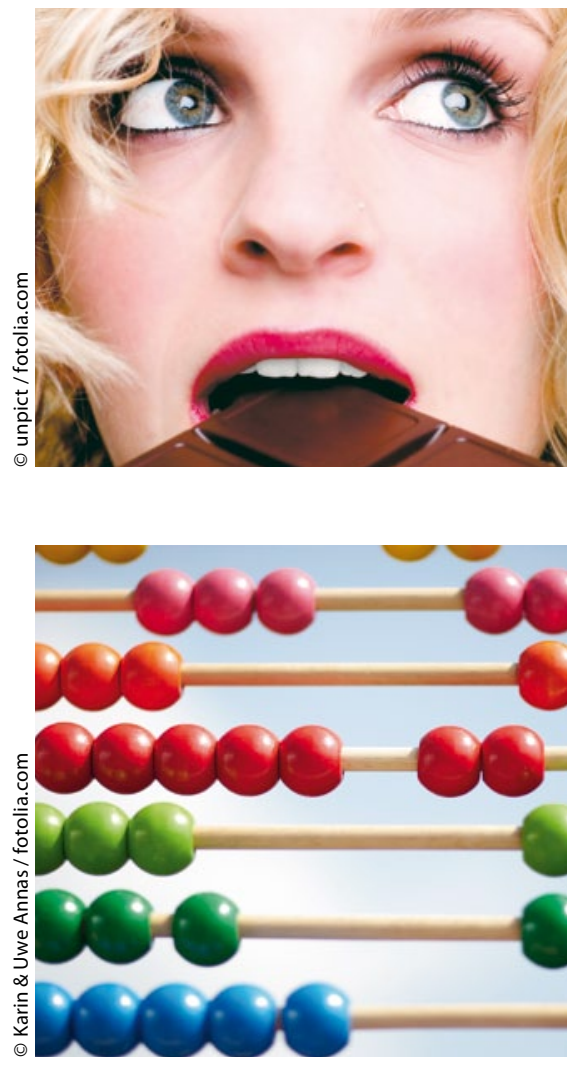

\title{
Diätetik in der Allergologie
}

- Nahrungsmittelallergien sind im wahrsten Sinne des Wortes in aller Munde. Nicht nur die einzelnen allergenen Komponenten spielen dabei eine entscheidende Rolle, sondern auch ihre Darreichungsform. Ob erhitzt oder im rohen Zustand - es kommt ganz entscheidend auf die Vorbehandlung und auf die Darreichungsformen an. Durch die Möglichkeit der Diskussion, die das Veranstaltungsformat AllergoActiv auszeichnet,

\section{1 x 1 der Immunologie}

— In Anlehnung an die Allergo-JournalSerie "Das kleine 1 x 1 der Immunologie", die von der Sektion Immunologie der DGAKI initiiert wurde, werden in der AllergoCompact-Veranstaltung "1 1 1 der Immunologie“ die grundlegenden Zusammenhänge aus dem Bereich der T-Zellen und Dendritischen Zellen dargestellt. Die besondere Bedeutung der angeborenen Immunität bei der Entwicklung von Allergien wird im Vortrag über die neutrophilen Granulozyten darge- kann diese sehr praxisrelevante Problematik von unterschiedlichen Seiten beleuchtet werden.

\section{AllergoActiv: Diätetik in der Allergologie: Geschüttelt oder gerührt? Hitzebehandlung und mehr}

Donnerstag, 5. September 2013, 16.00-17.00 Uhr und 17.00-18.00 Uhr

stellt. Insgesamt wird hier also eine kompakte Übersicht über die wichtigsten Zellen im Allergie- und Entzündungsgeschehen gegeben.

AllergoCompact: $1 \times 1 \mathrm{der}$ Immunologie

Freitag, 6, September 2013, 13.00-14.30 Uhr

\section{Zertifizierte Fortbildung im Allergo Journal}

Das Allergo Journal bietet Ihnen in jeder Ausgabe einen Fortbildungsbeitrag, der mit maximal drei CME-Punkten zur zertifizierten Fortbildung anerkannt ist. Als Zeitschriftenabonnent stehen Ihnen in der e.Akademie alle Fortbildungskurse Ihrer Zeitschrift als e.CME (Beitrags-PDF plus CME-Fragebogen) zur Verfügung. Darüber hinaus können Sie Kurse Ihrer Zeitschrift, deren Zertifizierungszeitraum abgelaufen ist, weiterhin für Ihre Fortbildung und einen persönlichen Wissenscheck nutzen.

\section{So einfach geht's:}

\section{Registrieren und einloggen}

Um Fortbildungseinheiten in der e.Akademie bearbeiten zu können, müssen Sie sich einmalig mit Ihrer Abonummer registrieren. Sind Sie bereits bei springermedizin.de registriert, können Sie unter Meine Daten > Mein Account > Abo hinzufügen ihre Abonummer hinterlegen. Sie finden diese auf Ihrem Adressetikett.

\begin{abstract}
2. Beitrag auswählen
e.Akademie > Kursübersicht $>$ Kurse meiner Fachzeitschriften auswählen und den gewünschten Kurs starten. Der Kurs kann jederzeit unterbrochen und später fortgesetzt werden.
\end{abstract}

3. CME-Punkte sammeln

Zu jedem Beitrag gehört ein Fragebogen mit zehn CMEFragen. Ab sieben richtigen Antworten haben Sie bestanden und erhalten umgehend eine Teilnahmebescheinigung!

\section{Teilnehmen und weitere Informationen unter:} springermedizin.de/eAkademie

Unser Tipp: Noch mehr Fortbildung bietet das e.Med-Komplettpaket. Hier stehen Ihnen in der e.Akademie alle Kurse der Fachzeitschriften von Springer Medizin zur Verfügung.

Testen Sie e.Med gratis und unverbindlich unter springermedizin.de/eMed 